\title{
Development of MR Compatible Surgical Manipulator toward a Unified Support System for Diagnosis and Treatment of Heart Disease
}

\author{
Fujio Tajima ${ }^{1}, K_{\text {Kousuke Kishi }}{ }^{1}$, Kouji Nishizawa ${ }^{1}$, Kazutoshi Kan ${ }^{1}$, \\ Yasuhiro Nemoto ${ }^{1}$, Haruo Takeda ${ }^{2}$, Shin-ichiro Umemura ${ }^{3}$, Hiroshi Takeuchi ${ }^{4}$, \\ Masakatsu G. Fujie ${ }^{5}$, Takeyoshi Dohi ${ }^{6}$, Ken-ichi Sudo ${ }^{7}$, and Shin-ichi Takamoto ${ }^{8}$ \\ ${ }^{1}$ Mechanical Engineering Research Laboratory, Hitachi, Ltd. \\ 502 Kandatsu, Tsuchiura-shi, Ibaraki 300-0013 Japan \\ ${ }^{2}$ Systems Development Laboratory, Hitachi, Ltd. \\ 1099 Ozenji, Asao, Kawasaki-shi, Kanagawa 215-0013 Japan \\ takeda@sdl.hitachi.co.jp \\ ${ }^{3}$ Central Research Laboratory, Hitachi, Ltd. \\ 1-280 Higashi-Koigakubo, Kokubunji-shi, Tokyo, 185-8601 Japan \\ sumemura@crl.hitachi.co.jp \\ ${ }^{4}$ Medical Systems Division, Hitachi, Ltd. \\ 1-5-1 Marunouchi, Chiyoda-ku, Tokyo, 100-8220 Japan \\ hi-takeuchi@med.hitachi.co.jp \\ ${ }^{5}$ Department of Mechanical Engineering, Waseda University \\ 3-4-1 Okubo, Shinjuku-ku, Tokyo, 169-8555 Japan \\ mgfujie@mn. waseda.ac.jp \\ ${ }^{6}$ Graduate School, Institute of Information Science and Engineering, Tokyo University \\ 7-3-1 Hongo, Bunkyo-ku, Tokyo, 113-8656 Japan \\ dohi@miki.pe.u-tokyo.ac.jp \\ ${ }^{7}$ School of Medicine, Kyorin University \\ 6-20-2 Shinkawa, Mitaka-shi, Tokyo, 181-8611 Japan \\ sudok@kyorin-u.ac.jp \\ ${ }^{8}$ School of Medicine, Tokyo University \\ 7-3-1 Hongo, Bunkyo-ku, Tokyo, 113-8656 Japan \\ takamoto-tho@h.u-tokyo.ac.jp
}

\begin{abstract}
We propose a new concept of a unified system for supporting both surgical treatment and intrasurgical diagnosis of heart diseases, especially ischemic heart disease like myocardial infarction, under a magnetic-resonanceimaging (MRI) environment. In developing the system, we first designed and built a prototype of maneuverable manipulator as a subsystem. We then evaluated MR compatibility of the manipulator by moving its arm tip close to a phantom in the field of view of an open-configuration MR imager. No noticeable deformation, but some signal-to-noise ratio (SNR) deterioration, was observed in the MR images taken during evaluation. It is planned to combine the manipulator with other subsystems and function modules in order to construct an easy-to-use unified support system. This system will then be applied to treat a variety of diseases of organs and tissues in the human body.
\end{abstract}




\section{Introduction}

Cardiovascular disease is the one of several major causes of death. Especially in Japan, it is said that one death out of four is caused by it, and ischemic heart disease like myocardial infarction accounts for some 50 percent of all cases of cardiovascular disease. This percentage is predicted to increase in the near future. Meanwhile, since the performance of various medical diagnosis devices, such as the magnetic resonance imager (MRI), has been making rapid progress over the last few years, it is no longer a dream that a surgeon can perform an operation while simultaneously watching images of multiple modalities acquired intrasurgically. To help make this dream a reality, we propose a unified system that supports intrasurgical diagnosis and minimally invasive surgery, mainly for ischemic heart disease, under MRI monitoring.

MRI has some advantages compared to conventional imaging devices like X-ray CT (computed tomography). Namely, it involves no exposure to radioactivity and can acquire tomographic images of a patient at any position from arbitrary orientation, and these imaging sequences provide many kinds of diagnostic information. Magnetic-resonance (MR)-compatible mechanisms for intervention and maneuverable surgical manipulator systems have been developed. Masamune [1] developed a needle-insertion manipulator for stereotactic neurosurgery in a conventional (i.e., not open-configured) MRI environment. It is a kind of stereotactic frame mechanism with motorized joints, and is composed of magnetic-insensitive materials like engineering plastics and metals like aluminum. In a collaborative research on an MR-compatible surgical robot system with an intraoperative MRI [2], Chinzei built a five-degree-offreedom (5DOF) tool-positioning manipulator. This system used a "double-donuts"type horizontal-magnetic-field open MRI, with a magnetic-field strength of 0.5 Tesla, manufactured by General Electric. The manipulator was maneuverable enough to hold surgical tools in a certain position and orientation.

Over the last several year research on manipulators that can perform surgical operations in the same way as a human hand has progressed considerably. Even commercial products have been clinically applied in many places in the world. The most popular one is called da Vinci (Intuitive Surgical) [3], which had FDA clearance for urology in June 2001 and clinical tests on cardiovascular surgery have been performed. Another is ZEUS (Computer Motion Inc.) [4]. These two products are socalled master-slave manipulator systems and they can perform very dexterous motion as an extention of a surgeon's hands. We have also been developing a multiple micromanipulator system that extends conventional stereotactic neurosurgery [5-7]. It enables an operator to make sub-millimeter manipulations that a surgeon's hand cannot do directly. We performed in-vivo operation experiments to improve the prototype system step by step. And it will be clinically tested in the near future. However, none of these manipulators referred to above are compatible with an MR environment (strong magnetic field and high-power radio-frequency waves). 


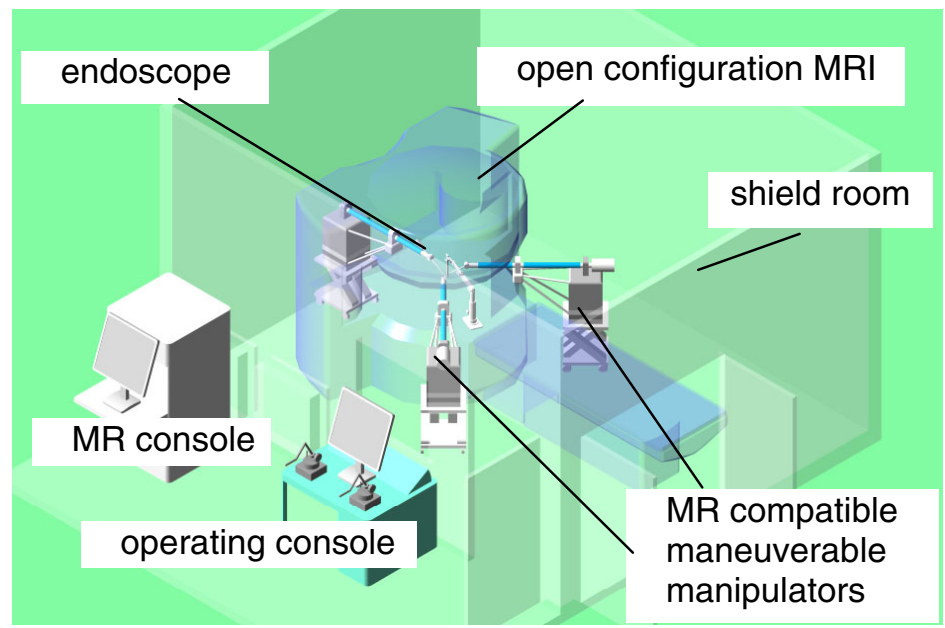

Fig. 1. Conceptual drawing of completed system

\section{Conceptual Design}

\section{System Design}

Figure 1 shows a conceptual drawing of the completed system. The system supports both diagnosis and treatment of cardiovascular disease under MR monitoring. It unifies image information from an optical endoscope, open MRI, an ultrasonic scanner, and conventional vital-sign detectors. Unified image information is updated periodically while surgery is being carried out. The updating period ranges from several tens of milliseconds to ten seconds, depending on the type of modalities. It helps and guides the surgeon and other medical staffs so they can make the right decisions. Moreover, the high-performance maneuverable manipulator can mimic the movement of the surgeon's hand precisely. The system uses a so-called master-slave control method in which an operation command through a master device is inputted and a slave manipulator moves according to the command almost instantly. The system also detects intrasurgical time-variant deformation of target organs by processing endoscopic images and information from a gap sensor attached at the distal end of the manipulator. The manipulator is then controlled so that the configuration of the arm tip in relation to the target point becomes appropriate for the operation. The manipulator mechanism is made of an insulator like ceramics or engineering plastics, or at least materials of low susceptibility to magnetism. This means that the MRI images do not suffer noise or distortion and that the manipulator is not affected by interference due to electromagnetic interaction. The actuators should not be electromagneticforce driven for the same reason, so ultrasonic motors are used instead.

An example of the system operation is explained as follows. The manipulators are located at the side of the bed under open MRI. Before the surgery, the medical staffs perform preparation procedures like anesthetization, sterilization, and skin cleaning on the patient. After that, the bed moves so that the target organ or tissue is within the 
field of view of the open MR imager. Surgeons carry out the operation by using the maneuverable manipulators with the support of the image-information system, which gives them multi-modal images from the endoscope, ultrasonic scanners, and the open MRI. The configuration of the endoscope and operation viewpoints are controlled, and the images presented are changed and arranged by user interfaces like foot pedals and a voice recognition unit.

\section{System Configuration and the Functions}

The conceptual image in Fig. 1 is translated into a schematic diagram shown in Fig. 2 , which shows how the total system works by presenting the subsystems and functional modules and the data flow among them. The system consists of three main parts: multimodal diagnostic devices, an intrasurgical information support system, and a maneuverable manipulator system. The functions of the developed subsystems are enclosed in bold-line rectangles and are summarized below:

(1) Surgical treatment using maneuverable manipulators compatible with MR;

(2) Shape-change compensation for surgical treatment without cardioplegia and cardiopulmonary support;

(3) MR-compatible fast 3D ultrasonic scanning;

(4) Image processing for various modalities into understandable forms;

(5) Image presentation of surgical guidance information, endoscopic images, and various information from multimodal diagnostic devices and vital-sign detectors in a manageable manner;

(6) Fine operation such as anastomosis.

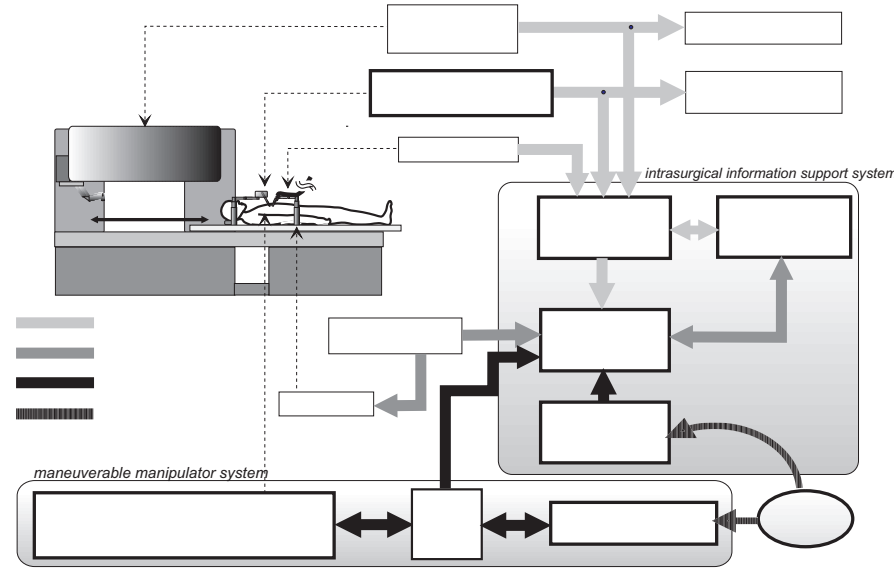

Fig. 2. Schematic diagram of the system

\section{Design of Manipu- lator Mechanism}

The maneuverable manipulator was designed so that a surgeon can perform MIDCAB (minimally-invasive direct coronary artery bypass), especially LITA (left internal thoracic artery)LAD (left anterior descending branch) anastomosis. Before the manipulator

mechanism was designed, preliminary studies were done on the motion of the surgeon during the operation.

First, motion and operation of a surgeon were analyzed. To do so, MIDCAB was decomposed into a series of operations, and it was found that anastomosis is usually required and can be done by a certain mechanical entity, namely, a maneuverable manipulator. 
Then, the anastomosis operation was split into a series of single motions and measured the approximate distance of movement and the volume of workspace during each motion. Next, the transition of the manipulator configuration at the distal end of the surgical tools was investigated in order to find how many degrees of freedom are needed in each single motion (further explanation is not given here.)

The above investigation was led to

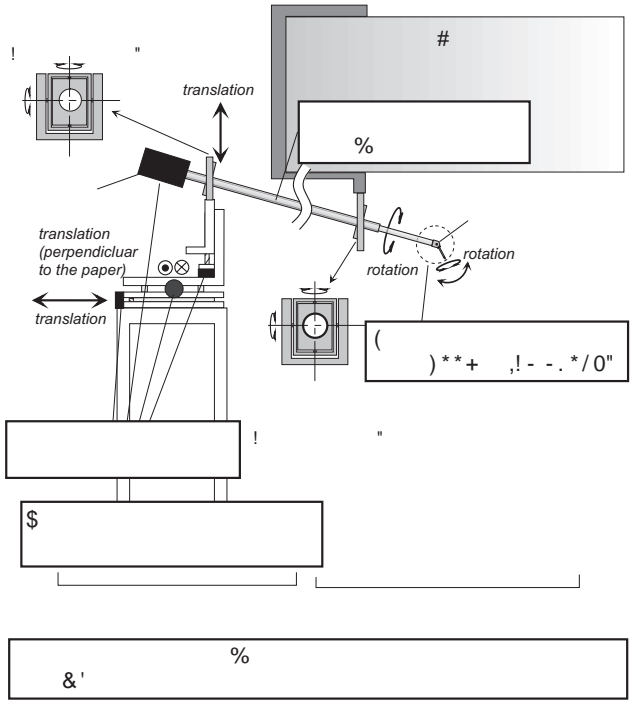

Fig. 3. Basic concept of manipulator mechanism the following guiding principles for the manipulator design in Fig. 3:

(1) The manipulator mechanism should be specialized for anastomosis (light-loaded, small workspace, dexterous);

(2) The base link should be placed apart from the open MRI magnet so as not to interfere with the MRI image, and the long arm (made of magnetically insusceptible materials) should cover the whole field of view;

(3) Number of degrees of freedom should be at least six;

(4) Working distance at the arm tip should be at most 40 to $50 \mathrm{~mm}$ for all kinds of single motion;

(5) Actuators must be concentrated at the proximal end (i.e., as far from the magnetic field as possible)

(6) Joints for translation and orientation should be located separately (former concentrated around the base, latter around the distal end);

(7) The arm should be exchangeable for sterilization and re-use.

\section{Implementation}

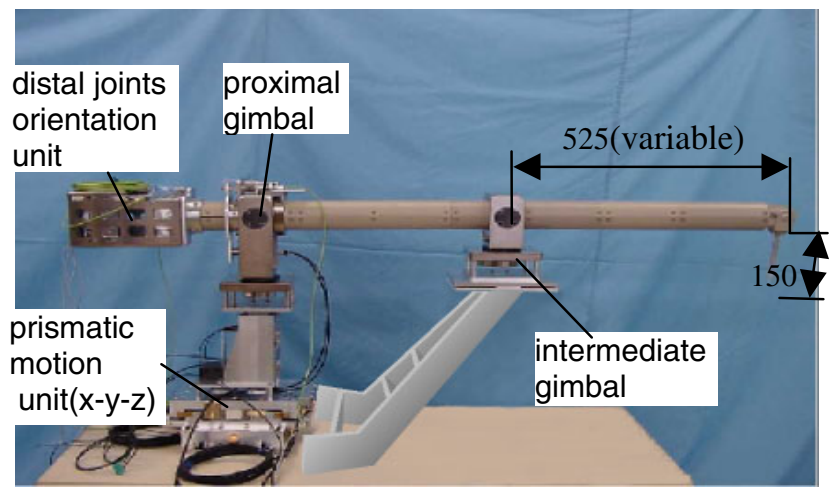

Fig. 4. Prototype of manipulator system
Figure 4 shows a view of the prototype of the manipulator. This manipulator has six degrees of freedom so that it can assume any configuration at its arm tip. Three prismatic motion units are fixed at its base. A motor at the proximal gimbal rotates the arm. The arm is supported and constrained by the 
intermediate gimbal to lessen deflection and vibration of the arm. The distal joints form a pair of differential gears, and the orientation of the arm tip is controlled by driving the gears with actuators at the proximal end through high-molecular-weight wires. Materials and components were selected according to preliminary examination under a 1.5 T MR environment. Especially at the arm tip, no damage to human tissue and the ability to withstand sterilization were considered beside MR safety. The main material of the prismatic motion units and gimbals was aluminum alloy. The arm and its distal joints were made of engineering plastics, mainly PEEK (poly-ether-etherketone). Two types of actuators were incorporated: an ultrasonic motor and a ceramic linear actuator. Position sensors were optical fiber rotary encoders and optical linear scales. These actuators and sensors were located a meter away from the center of the field of view, and no materials that are susceptible to magnetism incorporated were used. Light source and receiver of those position sensors were kept apart through optical fibers so that high power RF pulse emitted from the MRI does not affect sensor signals.

\section{Evaluation of MR Compatibility}

The manipulator was set up as shown in Fig. 5 to evaluate its effects on MR images. Preliminary studies were done under 1.5 T-MR environment (no details given here for want of space). A 0.3-T open configuration MRI (AIRIS II comfort, manufactured by Hitachi Medical Corporation, Japan) was used for imaging. Imaging sequences were spin echo (SE) and gradient field echo (GE). Imaging conditions are listed in the table 1.

Table 1. Imaging conditions

\begin{tabular}{l|c|c|c|c|c|c|c}
\hline item & TR & TE & $\begin{array}{c}\text { flip } \\
\text { angle }\end{array}$ & $\begin{array}{c}\text { number of } \\
\text { pixels }\end{array}$ & FOV & $\begin{array}{l}\text { slice } \\
\text { thickness }\end{array}$ & band width \\
\hline unit & {$[\mathrm{ms}]$} & {$[\mathrm{ms}]$} & {$[\mathrm{deg}]$} & WxH & {$[\mathrm{mm}]$} & {$[\mathrm{mm}]$} & {$[\mathrm{KHz}]$} \\
\hline \hline SE & 200 & 27 & - & $256 \times 256$ & 220 & 10 & 15.0 \\
\hline GE & 50 & 22.6 & 70 & $256 \times 256$ & 220 & 10 & 15.0 \\
\hline
\end{tabular}

A phantom was used in the tests. It is a polyethylene cylindrical vessel filled with $\mathrm{CuSO}_{4}$ solution (diameter: $150 \mathrm{~mm}$, height: $300 \mathrm{~mm}$ ). Images were taken while the manipulator was in motion. The manipulator repeatedly traced a rectangular trajectory at its arm tip between the narrow gap in the head coil.

Figure 6 shows MR images of the cylindrical phantom taken by SE and GE sequences. No severe artifacts were observed in the images, although the contrast in the image taken with GE sequence is deteriorated. These images were printed onto films usually used for diagnosis with the original data, and they were examined by clinicians. Their opinion was that they did not recognize any significant deformation even between the images taken with GE, and they are acceptable as long as they are used for intrasurgical survey, position tracking and the like, not for close diagnosis.

A quantitative comparison of contrast deterioration was done with signal-to-noise ratio (SNR) of each image, which is given by the definition:

$$
S N R=\operatorname{Smean} / S D,
$$




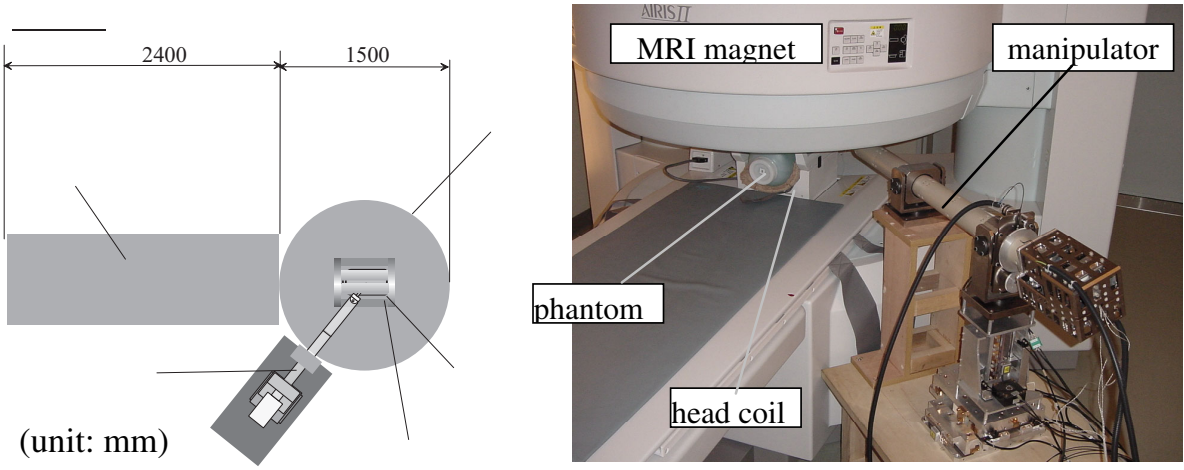

Fig. 5. Manipulator setting at an open configuration MRI

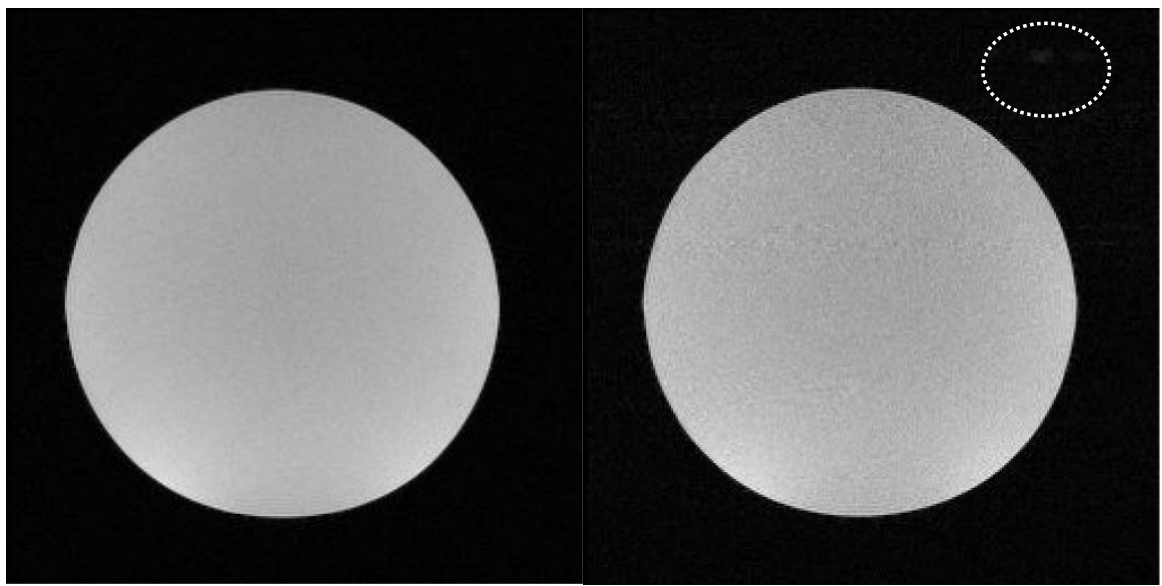

manipulator: NOT present

manipulator: in motion

(a) imaging sequence: spin echo

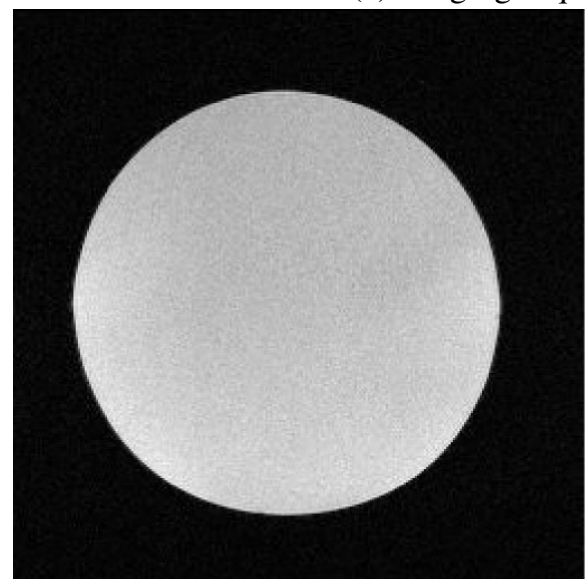

manipulator: NOT present

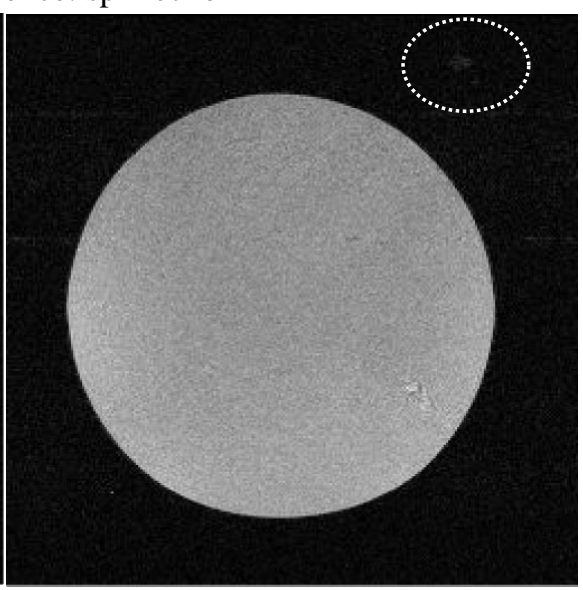

manipulator: in motion

(b) imaging sequence: gradient field echo

Fig. 6. MR images of a cylindrical phantom taken in two sequences. Dotted ellipses show the area where the tip of the manipulator moved. 
where Smean is the average intensity value of 240 pixels in a circle at the center of the image, and $S D$ is the average value of $S D n(n=1-4)$, which is the standard deviation of 60 pixels in a circle at each corner of the image. Figure 7 compares $S N R$ under several conditions. As a conventional method for comparing signal power, $S N R$ values are plotted on a logarithmic scale, that is, a decibel $\left(20 \log _{10} S N R\right)$ expression. As shown in Fig.6, SNR values were $38.3 \mathrm{~dB}$ (SE) and $33.4 \mathrm{~dB}$ (GE) when the manipulator was not present. They decreased to $33.6 \mathrm{~dB}(\mathrm{SE})$ and $27.0 \mathrm{~dB}(\mathrm{GE})$ when the manipulator was in motion.

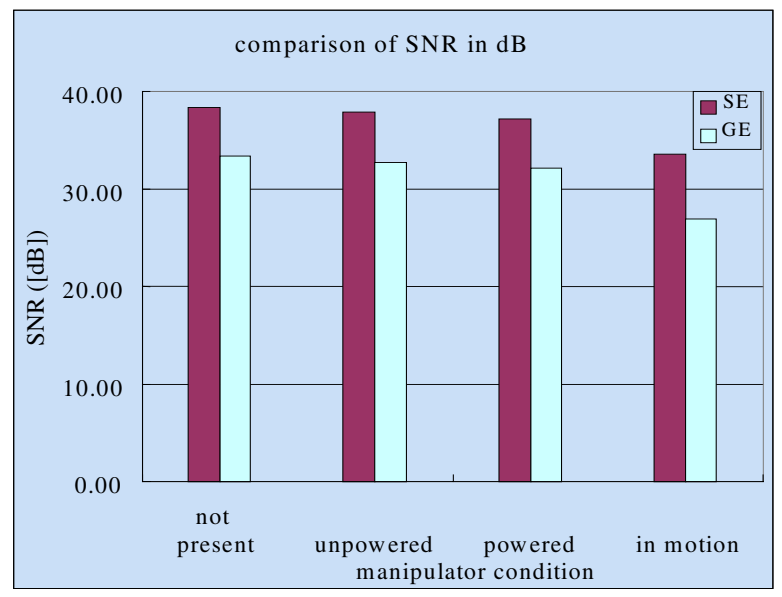

Fig. 7. Comparison of SNR in dB under 0.3-T open-configuration MRI

\section{References}

[1] Masamune, et al., "Development of a MRI Compatible Needle Insertion Manipulator for Stereotactic Neurosurgery", Proc. MRCAS, pp. 165-172 (1995)

[2] http://www.aist.go.jp/MEL/soshiki/kiso/biomech/chin/MMM/mrmani0-e.htm

[3] http://www.intuitivesurgical.com/html/daindex.html

[4] http://www.computermotion.com/zeus.html

[5] Kan, K. et al., "Microscopic Manipulator System for Minimally Invasive Neurosurgery", Proc. on the 12th International Symposium and Exhibition of Computer Assisted Radiology and Surgery (CAR'98), pp. 594-598 (1998)

[6] Kan, K. et al., "Development of HUMAN System with Three Micro Manipulators for Minimally Invasive Neurosurgery", Proc. on the 15th International Symposium and Exhibition of Computer Assisted Radiology and Surgery (CARS 2001), pp. 144-149 (2001)

[7] Hongo, K. et al., "Microscopic-manipulator system for minimally invasive neurosurgery Preliminary study for clinical application", Proc. on the 15th International Symposium and Exhibition of Computer Assisted Radiology and Surgery (CARS 2001), pp. 265-269 (2001) 